\title{
Protection against acute radiation-induced lung injury: A novel role for the anti-angiogenic agent Endostar
}

\author{
KAI ZHANG, SHENGHUI YANG, YING ZHU, ANWEI MO, DAN ZHANG and LI LIU \\ Cancer Center, Union Hospital, Tongji Medical College, Huazhong University \\ of Science and Technology, Wuhan, Hubei 430022, P.R. China
}

Received December 5, 2011; Accepted March 12, 2012

DOI: $10.3892 / \mathrm{mmr} .2012 .903$

\begin{abstract}
Radiotherapy is commonly used to treat thoracic malignancies, but often causes severe lung injury. Currently there are no effective protective strategies against radiation-induced lung injury (RILI). This study aimed to evaluate the ability of an angiogenesis antagonist, Endostar, against RILI, and the underlying mechanism in a mouse model. A total of 108 C57BL/6 female mice were randomized into 4 groups $(n=27)$ : i) control group; ii) Endostar group, animals were administered $7.75 \mathrm{ml} / \mathrm{kg}$ Endostar through intraperitoneal injection; iii) irradiation group, RILI was induced by exposing the animals to a single external irradiation on the thoraces (6 MV X-ray, $12 \mathrm{~Gy}$ ); and iv) irradiation plus Endostar group, animals were subjected to Endostar treatment and irradiation as in groups 2 and 3. A total of 3 animals from each of the 4 groups were sacrificed at 1, 6, 12, 24 and $72 \mathrm{~h}$ and at 2, 4, 8 and 24 weeks following treatment. Clinical signs and pathology of RILI were examined. The expression of transforming growth factor- $\beta 1$ (TGF- $\beta 1$ ) in lungs was analyzed by real-time quantitative polymerase chain reaction (RT-QPCR) and immunohistochemistry. Compared with the control group, irradiation induced evident interstitial edema and a significant increase in inflammatory cells in the lungs $(\mathrm{P}<0.05)$. Correlated with these changes, a notable increase in TGF- $\beta 1$ mRNA level and a robust increase in TGF- $\beta 1$ immunoreactivity were observed in lung tissues in a time-dependent manner following irradiation $(\mathrm{P}<0.05)$. Endostar administration effectively attenuated the magnitude of the increase in inflammatory cells as well as the elevation of TGF- $\beta 1$ expression in lung tissues after RILI $(\mathrm{P}<0.05)$. In conclusion, radiation induced an increased expression of the inflammatory mediator TGF- $\beta 1$ and the associated pathogenesis in the lung, while Endostar was able to at least partially attenuate RILI through downregulating the expression of
\end{abstract}

Correspondence to: Professor Li Liu, Cancer Center, Union Hospital, Tongji Medical College, Huazhong University of Science and Technology, 156 Wujiadun, Jianghan, Wuhan, Hubei 430022, P.R. China

E-mail: liulixiehe2004@163.com

Key words: radiation-induced lung injury, transforming growth factor- $\beta 1$, Endostar, radiation protection
TGF- $\beta 1$ in mice. Our findings suggest that Endostar may be a novel protective agent against RILI.

\section{Introduction}

Pulmonary radiotherapy is an effective curative and palliative option for patients with malignant thoracic cancer. However, radiation-induced lung injury (RILI) remains a significant clinical toxicity from thoracic radiation with poor prognosis (1-3), thus limiting the dose of radiation delivered to tumors. Histopathologically, damage to endothelial or epithelial cells is assumed to be the initial step of RILI, which can result in pneumonitis and ultimately pulmonary fibrosis (4). Irradiation pneumonitis is characterized by edema of the interstitium and exudation into air spaces, infiltration of inflammatory cells, the loss of type I pneumocytes, increase in capillary permeability, and thickening of the alveolar septa $(5,6)$. Pulmonary fibrosis is characterized by the progressive fibrosis of alveolar septa and capillaries causing spread and obliteration of the residual alveoli and the lumina of the capillaries, which is considered an outcome of the repair mechanisms triggered by radiation pneumonitis. However, despite an extensive research effort in the past decades, the molecular events underlying the development of RILI remain unclear.

A number of pro-inflammatory cytokines and growth factors have been demonstrated to contribute to the pathogenesis of RILI (7-10,11). Among these, transforming growth factor- $\beta 1$ (TGF- $\beta 1$ ) is thought to play a crucial role $(10,12,13)$. TGF- $\beta 1$ is usually released from platelets at the site of a wound, where it recruits monocytes and macrophages, inhibits the proliferation of epithelial cells, enhances the maturation of fibroblasts into postmitotic fibrocytes resulting in production of fibrous tissue, promotes angiogenesis, and inhibits degradation of the extracellular matrix (14). In addition to its role in wound healing, TGF- $\beta 1$ has been detected in elevated levels in the lung following irradiation (8).

Agents, including glucocorticoid, angiotensin-1-converting enzyme inhibitors and non-steroidal anti-inflammatory drugs, are commonly applied for the treatment of RILI, however, their effectiveness has not met satisfactory levels and the side effects are significant (15). Endostatin, a proteolytic fragment of collagen XVIII, is a naturally occurring endogenous potent inhibitor of angiogenesis (16). It has been proven that endostatin can effectively suppress endothelial cell proliferation and 
migration in vitro (17), and inhibit tumor growth in various types of animal models in vivo (18). However, purification of endogenous endostatin is time-consuming, and production of the soluble type of recombinant endostatin in a yeast system has a low yield and high cost, therefore significantly limiting extensive clinical use of this anti-angiogenic agent (19).

Endostar is a novel recombinant analogue of human endostatin, which is expressed and purified in E. coli, and has an additional nine-amino acid sequence (MGGSHHHHH) at the N-terminal and a poly-histidine (six) tag. The modified protein has a longer half-life and higher affinity than endostatin $(19,20)$. It was first approved by the State Food and Drug Administration in 2005 for the treatment of non-small cell lung cancer in China (21). Pre-clinical studies have demonstrated that Endostar is capable of enhancing the response of human nasopharyngeal carcinoma and lung adenocarcinoma xenografts to radiotherapy in nude mice $(22,23)$.

Given the involvement of TGF- $\beta 1$ in the pathogenesis of RILI and the synergy between Endostar and radiation in treating malignant tumors, we hypothesize that Endostar may possibly protect normal lung tissue against thoracic radiationinduced injury through modulating the expression of TGF- $\beta 1$. This study aimed to test this hypothesis in a mouse model of thoracic irradiation.

\section{Materials and methods}

Animals. Female C57BL/6 mice, 8 weeks old and weighing $20 \pm 2$ g, were purchased from the Research Animal Facility of Tongji Medical College (Wuhan, China). They were housed in groups of 4-6 per cage in laminar flow hoods in a pathogen-free environment $\left(22 \pm 2^{\circ} \mathrm{C}, 55 \pm 10 \%\right.$ humidity and 12-12 h/light-dark cycle) with free access to a standard laboratory diet and water. The animals were acclimatized for 1 week prior to the experiments. The study protocol was reviewed and approved by the Medical Sciences Animal Care and Use Committee of the Huazhong University of Science and Technology.

Treatment allocations. Animals were randomized into: i) irradiation group, animals received a single dose of irradiation and multiple doses of normal saline $(7.75 \mathrm{ml} / \mathrm{kg}$ per day) through intraperitoneal injection; ii) Endostar group, animals received sham irradiation once and multiple doses of Endostar (7.75 ml/kg per day, $0.3 \mathrm{mg} / \mathrm{ml}$; Simcere Pharmaceutical Research, Nanjing, China) through intraperitoneal injection; iii) irradiation plus Endostar group, animals received a single dose of irradiation as in group 1 and Endostar as in group 2; iv) control group, animals received sham irradiation as in group 2 and normal saline as in group 1 . The initial administration of Endostar and normal saline was performed $2 \mathrm{~h}$ prior to irradiation or sham irradiation. A total of 3 animals from each of the 4 groups were sacrificed by cervical dislocation at each of the times (1, 6, 24, $72 \mathrm{~h}$ and 2, 4, 8 and 24 weeks) following irradiation or sham irradiation for analyses described below. The drug (Endostar) or sham (normal saline) treatment were administered daily until sacrifice at the corresponding times.

Irradiation was performed as previously described (5). The animal was briefly restrained with a plastic jig, and a single dose of $12 \mathrm{~Gy}$ (10 MV photons of beam energy at $2.4 \mathrm{~Gy} / \mathrm{min}$ ) was administered to the lung area $(18 \mathrm{~cm} \times 10 \mathrm{~cm})$ at a source surface distance (SSD) of $1 \mathrm{~m}$ while the head and abdomen were shielded with lead strips. The relative dose, location and area size of the thoracic irradiation were determined based on the ADAC Pinnacle Three-dimensional Treatment Planning System. Similar procedures were applied to animals designated to receive sham irradiation, but the irradiation area in the lung was completely shielded with lead blocks. Both irradiation and sham irradiation were performed under ketamine $(67.5 \mathrm{mg} / \mathrm{kg})$ and xylazine $(4.5 \mathrm{mg} / \mathrm{kg})$ anesthesia.

Tissue collection and initial processing. After sacrifice of the animals at the specific times, intact lungs were obtained. The left lobes were fixed in $10 \%$ neutral-buffered formaldehyde, embedded in paraffin and cut into $4-\mu \mathrm{m}$ sections. The tissue sections were mounted onto slides and stained for histological and immunohistochemical analyses. The right lung lobes were snap-frozen in liquid nitrogen for RNA isolation and subsequent real-time quantitative polymerase chain reaction (RT-QPCR).

Hematoxylin and eosin staining. Tissue sections were deparaffinized in xylene and re-hydrated in a series of alcohol solutions. After a brief wash in distilled water, the sections were stained in Harris hematoxylin solution for $5 \mathrm{~min}$, washed in tap water and counterstained in eosin-phloxine solution for $2 \mathrm{~min}$.

Masson staining. Paraffin-embedded tranverse sections were cut $(4 \mu \mathrm{m})$ and stained using Masson Trichrome. Lung injury was assessed by the manifestation of alveolitis and fibrosis. The severity of alveolitis and fibrosis was semi-quantitated using the score-grade system as described by Szapiel et al (24): absence of alveolitis (-, score 0 , grade 0 ); mild alveolitis with widened alveolar septum due to cell infiltration and the lesioned tissues accounted for $<20 \%$ of total lung $(+, 1,1)$; moderate alveolitis with lesions in $20-50 \%$ of the pulmonary tissue $(++, 2,2)$; severe alveolitis with lesions in $>50 \%$ of the pulmonary tissue $(+++, 3,3)$.

RT-QPCR analysis. Total RNA was extracted from frozen pulmonary tissue using TRIzol reagent (Invitrogen, Shanghai, China) according to the manufacturer's instructions. Oligo (dT18)-primed first-strand cDNA was synthesized using a First-Strand cDNA Synthesis kit (Invitrogen) according to the manufacturer's instructions. TGF- $\beta 1$ was amplified from the RT reaction mixture by 30 cycles of PCR $\left(30 \mathrm{sec}\right.$ at $94^{\circ} \mathrm{C}$, $40 \mathrm{sec}$ at $57^{\circ} \mathrm{C}, 40 \mathrm{sec}$ at $72^{\circ} \mathrm{C}$ and, finally, $5 \mathrm{~min}$ at $72^{\circ} \mathrm{C}$ ), followed by one cycle of denaturation of $4 \mathrm{~min}$ at $94^{\circ} \mathrm{C}$. A $\beta$-actin fragment was simultaneously amplified in the same reaction as an internal control. The primer sequences were as follows: TGF- $\beta 1$ sense, 5 '-ATC CTG TCC AAA CTA AGG CTC G-3' and anti-sense, 5'-ACC TCT TTA GCA TAG TAG TCC GC-3'; $\beta$-actin sense, 5'-AGA GGG AAA TCG TGC GTG AC-3' and anti-sense, 5'-CAA TAG TGA TGA CCT GGC CGT-3'. PCR products (TGF- $\beta 1,167 \mathrm{bp}$ and $\beta$-actin fragments, $138 \mathrm{bp}$ ) were visualized by ethidium bromide following electrophoresis on a $1 \%$ agarose gel, and quantitated by densitometry using a dual-intensity transilluminator equipped with Gel-Pro Analyzer version 3.1 (Media Cybernetics, Bethesda, MD, USA). The relative level of TGF- $\beta 1 \mathrm{mRNA}$ was expressed 
A

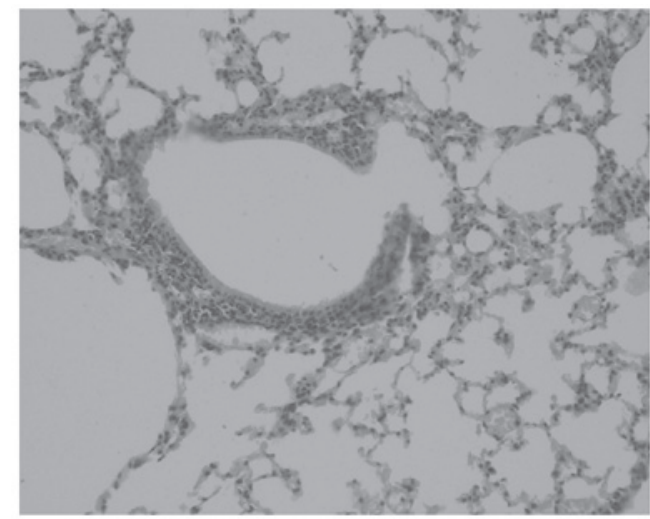

C

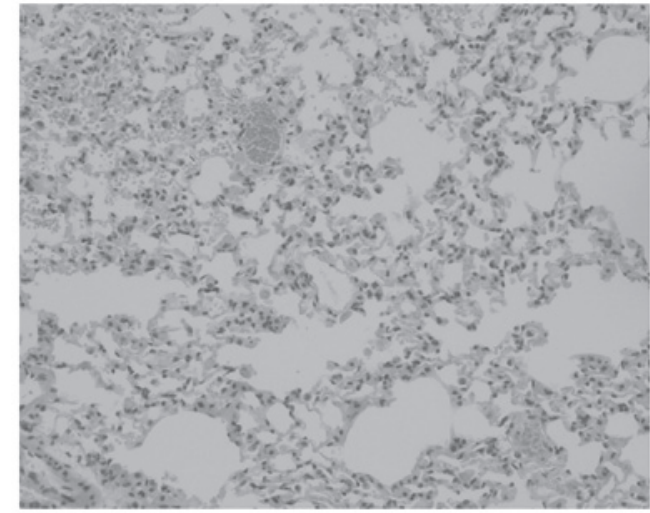

B

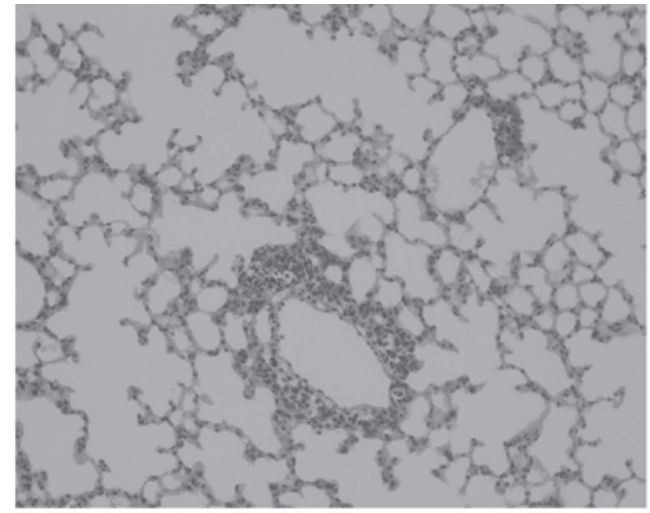

D

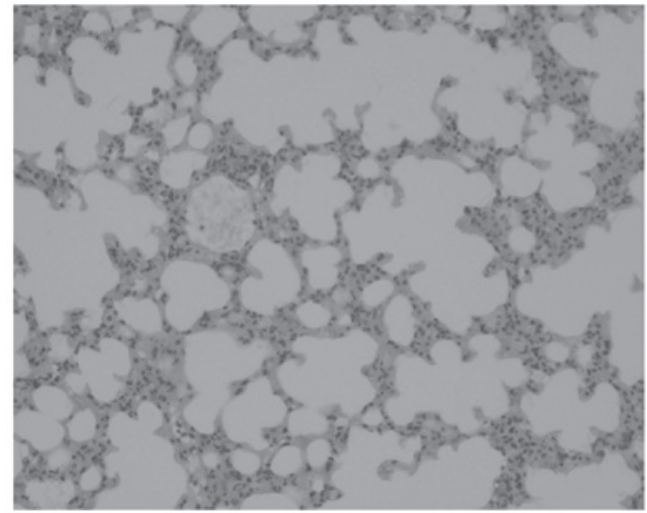

Figure 1. Digital images (x200) of pulmonary tissue sections following hematoxylin and eosin staining. (A) No pathology was observed in the normal control and (B) Endostar-treated non-irradiated mice. In contrast, pulmonary lesions, including alveolitis characterized by edema on the alveolar wall, hyperemia, widened alveolar septa and inflammatory cell infiltration, were evident in the (C) irradiated animals, but (D) the severity of the lesions was reduced in the Endostar-treated irradiated animals.

as the ratio of the densitometric value of the TGF- $\beta 1$ band over that of the $\beta$-actin band.

Immunohistochemistry. Tissue sections were blocked in $3 \% \mathrm{H}_{2} \mathrm{O}_{2}$ at $37^{\circ} \mathrm{C}$ for $10 \mathrm{~min}$ and incubated with the rabbit anti-mouse TGF- $\beta 1$ antibody $(1: 100, \mathrm{R} \& \mathrm{D}$ Systems, Minneapolis, MN, USA) at $4^{\circ} \mathrm{C}$ overnight, following deparaffinization, rehydration and dehydration. The following day, the sections were incubated with biotinylated anti-rabbit antibody (1:200, Invitrogen, CA, USA) at $37^{\circ} \mathrm{C}$ for $1 \mathrm{~h}$, and then with the avidin-biotin-peroxidase complex (ABC Complex; Dako, Glostrup, Denmark) for $30 \mathrm{~min}$ at room temperature. Following a final washing in PBS, TGF- $\beta 1$ signals were visualized with a DAB kit (Sigma, St. Louis, MO, USA), counterstained with haematoxylin and mounted in Kayser's glycerine gelatine. PBS replaced the primary antibody to serve as a negative control. TGF- $\beta 1$ immunoreactivity was quantified under a microscope using a semi-quantitative scoring method as previously described (26): 0, no immunostaining; 1, weak (light yellow); 2, moderate (yellow-brown); and 3, strong (brown). A score $>3$ was defined as a positive immune response. A total of 5 random fields were observed for each specimen and the scores were averaged for each time and treatment combinations. This analysis was repeated twice by an investigator blinded to the specimen identification.

Statistical analysis. The TGF- $\beta 1 \mathrm{mRNA}$ levels and TGF- $\beta 1$ protein expression (immunoreactivity in the lung paren- chyma, expressed as positive cell counts) were analyzed by one-way analysis of variance (ANOVA) followed by Bonferroni test for multiple comparisons. SPSS 17.0 statistical software was used. $\mathrm{P}<0.05$ was considered to indicate a statistically significant difference.

\section{Results}

Histological changes. Pulmonary alveolitis was not detected in mice in the control and Endostar treatment groups, but was evident in mice in the irradiation and irradiation plus Endostar treatment groups at various times, as demonstrated by hematoxylin and eosin staining (Fig. 1). Alveolitis was characterized by macrophage infiltration into the air spaces, edema of the alveolar wall, desquamation of epithelial cells from the alveolar walls, hyperemia, thickening of the alveolar septa by infiltration of inflammatory cells, collagen deposition, progressive fibrosis and obliteration of the alveoli. Focal fibrosis was clearly observed within the inflammation region 24 weeks after irradiation. The mean grade of alveolitis was 1-2 in the irradiation plus Endostar treatment group and was 2-3 in the irradiation group; the difference was significant $(\mathrm{P}<0.05)$.

To further confirm fibrosis, Masson staining was performed. The results demonstrated that irradiation induced fibrosis, with accumulation of fibroblasts and deposition of collagen around blood vessels and in the interstitial region in the lung, and Endostar partially ameliorated the irradiation-induced fibrosis (Fig. 2). 
A

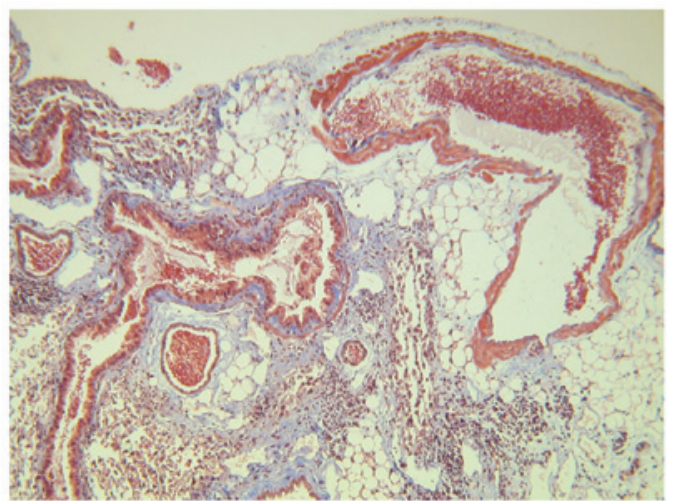

B

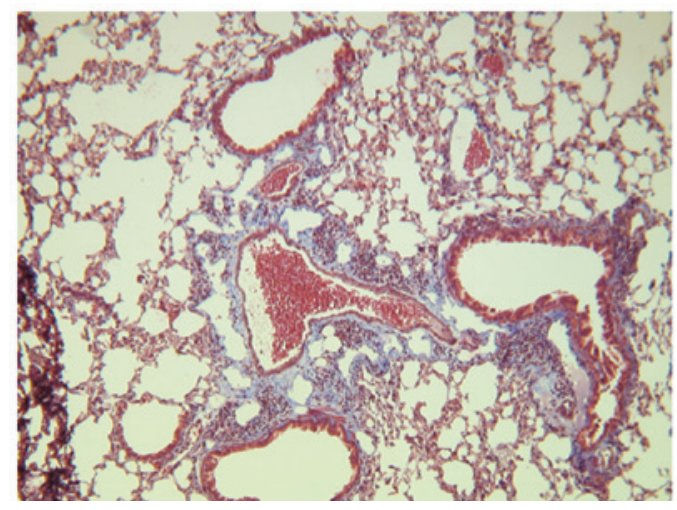

Figure 2. Digital images (x100) of pulmonary tissue sections following Masson staining. (A) At 24 weeks, fibrosis characterized by accumulation of fibroblasts, deposition of collagen around blood vessels and in the interstitial region was evident in the irradiated animals, (B) but the severity was reduced in the Endostar-treated irradiated mice.

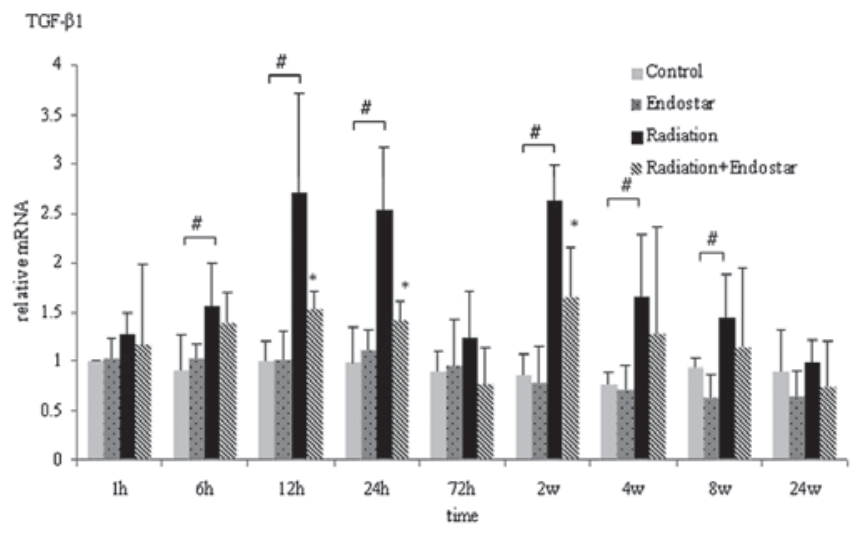

Figure 3. Relative TGF- $\beta 1$ mRNA levels (mean $\pm \mathrm{SD}$ ) in different groups of animals at various times. "Statistically significant differences between the radiation and radiation plus Endostar groups. "Statistically significant differences between the radiation and sham-radiation groups.

Changes in TGF- $\beta 1$ mRNA level. The data on the relative levels of TGF- $\beta 1$ mRNA in the lungs of mice in the different groups are presented in Fig. 3. Compared with the control, Endostar alone did not alter the TGF- $\beta 1$ mRNA abundance at any of the times studied $(\mathrm{P}>0.05)$, but thoracic irradiation elevated TGF- $\beta 1$ mRNA level in a time-dependent manner. Treatment of the irradiated animals with Endostar significantly attenuated the irradiation-induced elevation of TGF- $\beta 1$ mRNA level at $12,24 \mathrm{~h}$ and 2 weeks after irradiation $(\mathrm{P}<0.05)$, but not at 1 , 6 and $72 \mathrm{~h}$ and 4, 8, 24 weeks after irradiation; $\mathrm{P}>0.05$ ).

Changes in TGF- $\beta 1$ immunoreactivity. Fig. 4 shows digital images demonstrating TGF- $\beta 1$ histoimmunochemical staining signals on the pulmonary tissue sections prepared from animals in the different experimental groups at various times. Significant increases in TGF- $\beta 1$ expression were observed at every time post-irradiation for all radiation groups compared to the control and Endostar groups. In the control group, positive staining was restricted to the bronchiolar epithelium, while TGF- $\beta 1$ staining obtained 4 weeks after irradiation demonstrated strong immunostaining of the bronchial epithelial cells and interstitial inflammatory cells. Treatment with Endostar attenuated the expression of
TGF- $\beta 1$ in the lung tissue post-irradiation. The immunohistochemical scores of TGF- $\beta 1$ varied with time (Fig. 5). The TGF- $\beta 1$ score peaked at 4 weeks post-irradiation. Although the changes in TGF- $\beta 1$ expression followed the same trend in the radiation plus Endostar and radiation groups, the former had significantly lower levels of TGF- $\beta 1$ expression than the latter at 2, 4 and 8 weeks after irradiation (Fig. 5; P<0.05).

\section{Discussion}

The human lung is among the most sensitive and critical tissue of concern in local and systemic radiation exposure. Lung injuries are frequently observed in patients who have undergone thoracic irradiation for the treatment of lung, breast or hematologic malignancies. Radiation-induced damage to normal lung tissue remains the dose-limiting factor in chest radiotherapy. Therefore, preclinical research in animal models, aimed to uncover the underlying mechanism of RILI and evaluate mitigating therapies, is urgently required (25). In the present study, using mice as a model, we investigated the involvement of TGF- $\beta 1$ in the pathogenesis of RILI, and the novel role of Endostar in preventing RILI.

We first assessed morphopathological changes in the lung by histochemical and Masson staining. The results clearly demonstrated pulmonary lesions characterized by alveolitis and pneumonitis with inflammatory cell accumulation and collagen deposition in the irradiated mice (Fig. 1). Cellular interactions between lung parenchymal cells and circulating immune cells have been suggested as a mechanism underlying the pathogenesis of RILI (6,26-28). Results from our morphopathological analysis support this mechanism.

We next assessed the changes in TGF- $\beta 1$ expression in the lung, in order to attempt to understand the role of this cytokine in the pathogenesis and recovery of RILI. There is increasing evidence suggesting that the development of certain types of radiation injury may be mediated through the Smad-independent TGF- $\beta 1$ signaling pathway $(29,30)$. It has been identified that binding of TGF- $\beta 1$ to its type II transmembrane receptor results in the subsequent formation of a complex with the type II receptor. Upon phosphorylation, this complex activates the signaling proteins Smad2 and Smad3. The activated Smad2 and 3 proteins then bind to 
A

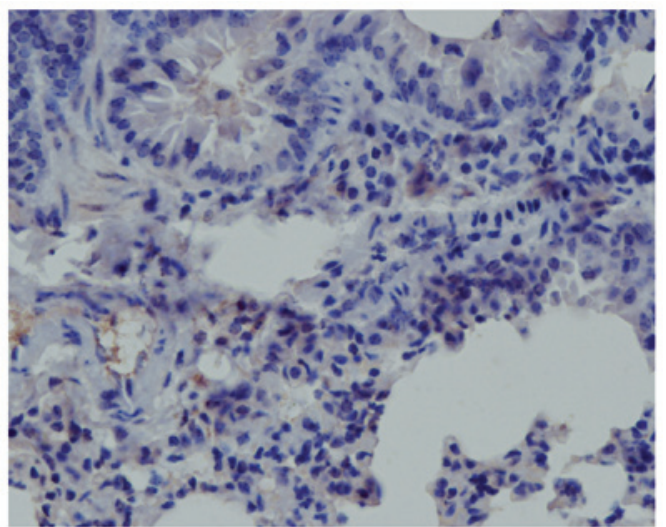

C

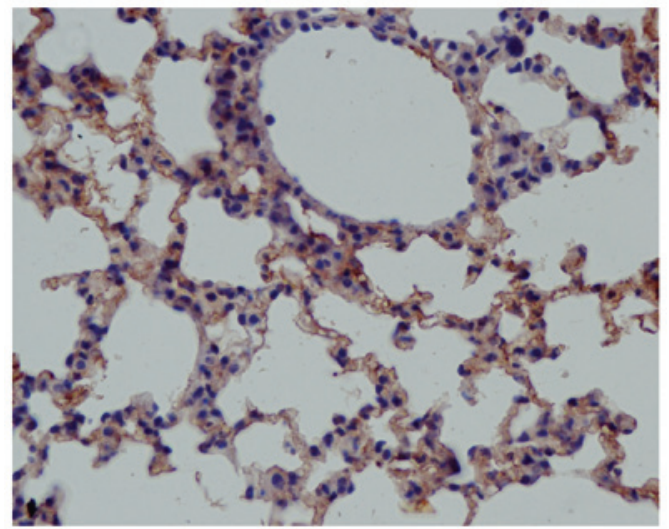

E

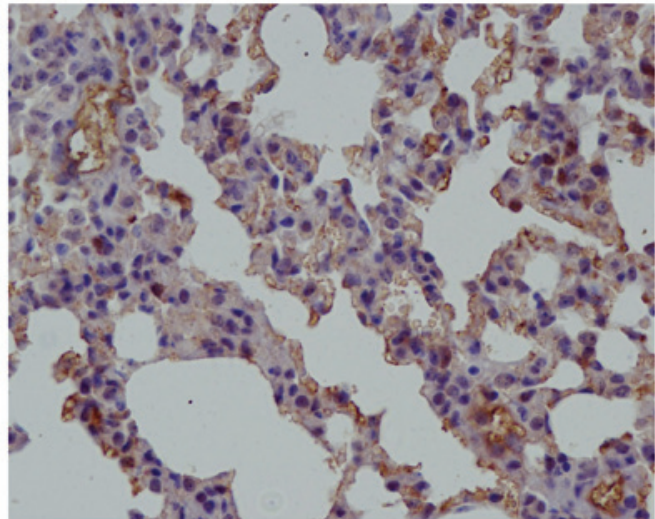

G

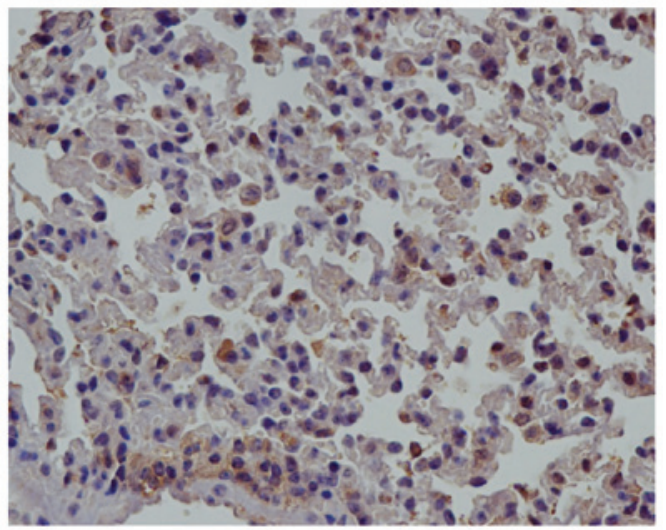

Smad4 and translocate to the cell nucleus, where they modulate downstream target gene transcription through binding to the response elements on the promoters of target genes (14). In the present study, changes in the expression of TGF- $\beta 1$ in the lung were analyzed at mRNA and protein levels through
B

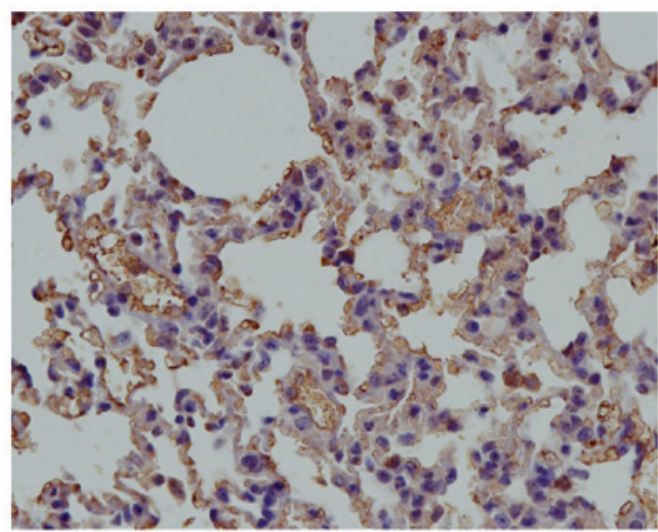

D

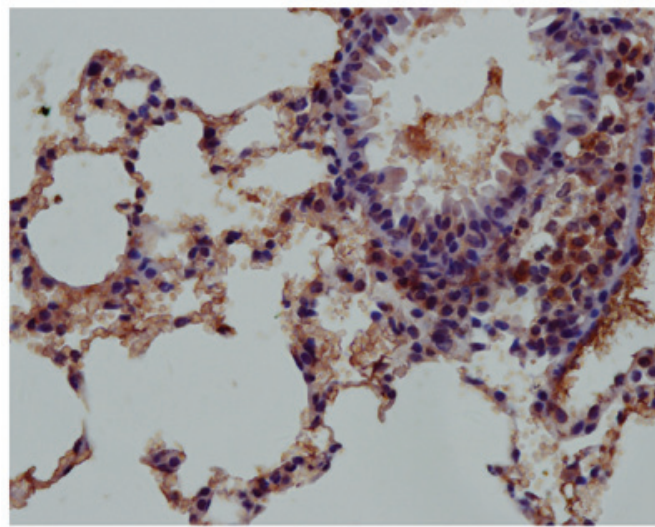

$\mathbf{F}$

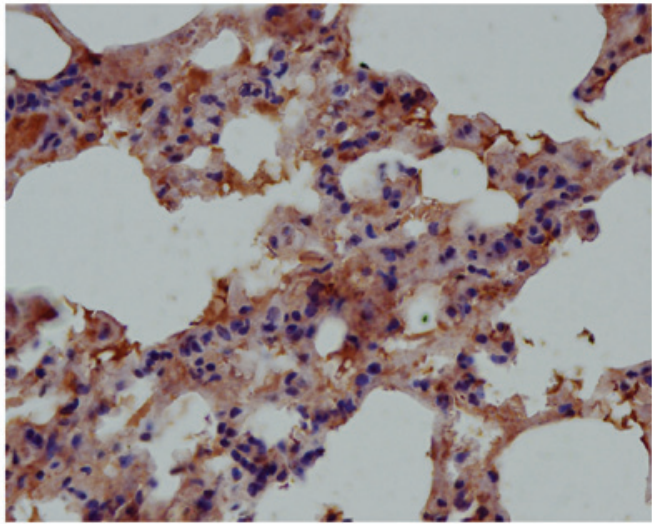

Figure 4. Digital images (x400) of pulmonary tissue sections after TGF- $\beta 1$ immunohistochemical staining. (A) Control animals, (B) animals after 2 weeks of irradiation, (C) Endostar-treated animals after 2 weeks of irradiation, (D) animals after 4 weeks of irradiation, (E) Endostar-treated animals after 4 weeks of irradiation, (F) animals after 8 weeks of irradiation, (G) Endostar-treated animals after 8 weeks of irradiation.

real-time PCR and immunostaining, respectively. The results demonstrated that TGF- $\beta 1$ mRNA levels and immunostaining intensity in the lung increased in a time-dependent manner; detectable event $1 \mathrm{~h}$ after thoracic irradiation, significant from post-irradiation hour 6 onwards and sustained to the end of 


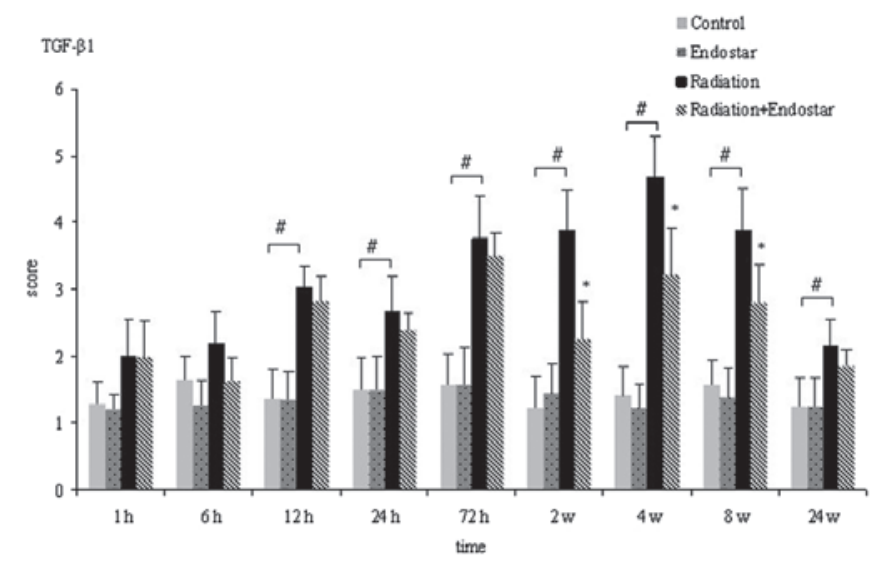

Figure 5. Average scores of TGF- $\beta 1$ immunohistochemical staining in different groups at various time points. Data are the mean \pm SD of triplicate determinations using three mice. *Statistically significant differences between the radiation and radiation plus endostar groups. "Statistically significant differences between the radiation and sham-radiation groups.

the experiment ( 24 weeks). This pattern of change in TGF- $\beta 1$ expression in the lung, associatied with the development of RILI, is consistent with the pattern observed in previous in vivo studies in mice and rats $(5,31,32)$.

Endostar is an analogue of endostatin, a naturally occurring endogenous inhibitor of neovascularization, which has been widely used in anti-angiogenesis therapy for cancer $(21,33)$. Recently, it has been demonstrated that endostatin can synergistically increase the sensitivity of tumors to radiotherapy (34). These findings advocated the evaluation of the value of Endostar in protection against thoracic irradiation-induced adverse effects. Through histological, RT-QPCR and immunohistochemical analyses, we demonstrated that the pulmonary morphology and TGF- $\beta 1$ mRNA abundance and immunoreactivity in the pulmonary tissue in animals of the Endostar treatment group were similar to those in the normal control animals. It was also revealed that pulmonary alveolitis, edema, inflammation and fibrosis were less severe with decreased expression of TGF- $\beta 1$ in the irradiated animals that received Endostar treatment compared to those irradiated animals that did not receive Endostar treatment. These observations clearly suggest that Endostar neither adversely affected the pulmonary morphology nor significantly changed the pulmonary expression of TGF- $\beta 1$, but effectively protected the lung from severe irradiation-induced injury. Although the mechanism underlying the protection of Endostar against RILI require further investigation, our findings suggest that protection may involve molecular cascades mediated by profibrogenic agents and proangiogenic cytokines. In accordance with this hypothesis, Tanabe et al (35) reported that administration of endostatin significantly increased expression of VEGF-A, $\alpha$-SMA and profibrotic TGF- $\beta 1$, and supressed peritoneal sclerosis in a dose-dependent manner in a mouse model. Vujaskovic et al (36) and Gauter-Fleckenstein et al (37) further demonstrated that profibrinogenic agents, including TGF- $\beta 1$ and proangiogenic cytokines, were involved in the pathogenesis of RILI, while Jackson et al (38) demonstrated that radiation injury was associated with hypoxia-induced increases in macrophage infiltration/ activation and TGF- $\beta$ and VEGF production.
In summary, the present mouse study demonstrated that irradiation-induced TGF- $\beta$ upregulation-associated pulmonary lesions and the anti-angiogenic agent, Endostar, was able to effectively alleviate the severity of irradiation-induced pulmonary lesions. These novel findings suggest that Endostar has great potential to be used in treating malignant cancer patients to protect from radiotherapy-induced lung injury in clinical settings.

\section{References}

1. Marks LB, Yu X, Vujaskovic Z, Small W Jr, Folz R and Anscher MS: Radiation-induced lung injury. Semin Radiat Oncol 13: 333-345, 2003.

2. Mehta V: Radiation pneumonitis and pulmonary fibrosis in non-small-cell lung cancer: pulmonary function, prediction, and prevention. Int J Radiat Oncol Biol Phys 63: 5-24, 2005.

3. Kong FM, Hayman JA, Griffith KA, Kalemkerian GP, Arenberg D, Lyons S, Turrisi A, Lichter A, Fraass B, Eisbruch A, Lawrence TS and Ten Haken RK: Final toxicity results of a radiation-dose escalation study in patients with non-small-cell lung cancer (NSCLC): predictors for radiation pneumonitis and fibrosis. Int J Radiat Oncol Biol Phys 65: 1075-1086, 2006.

4. Rübe CE, Wilfert F, Uthe D, Schmid KW, Knoop R, Willich N, Schuck A and Rübe C: Modulation of radiation-induced tumor necrosis factor alpha (TNF-alpha) expression in the lung tissue by pentoxifylline. Radiother Oncol 64: 177-187, 2002.

5. Rübe CE, Uthe D, Schmid KW, Richter KD, Wessel J, Schuck A, Willich N and Rübe C: Dose-dependent induction of transforming growth factor beta (TGF-beta) in the lung tissue of fibrosis-prone mice after thoracic irradiation. Int J Radiat Oncol Biol Phys 47: 1033-1042, 2000.

6. Dong XR, Wang JN, Liu L, Chen X, Chen MS, Chen J, Ren JH, Li Q and Han J: Modulation of radiation-induced tumor necrosis factor- $\alpha$ and transforming growth factor $\beta 1$ expression in the lung tissue by Shengqi Fuzheng injection. Mol Med Report 3: 621-627, 2010.

7. Brush J, Lipnick SL, Phillips T, Sitko J, McDonald JT and McBride WH: Molecular mechanisms of late normal tissue injury. Semin Radiat Oncol 17: 121-130, 2007.

8. Rübe CE, Uthe D, Wilfert F, Ludwig D, Yang K, König J, Palm J, Schuck A, Willich N, Remberger K and Rübe C: The bronchiolar epithelium as a prominent source of pro-inflammatory cytokines after lung irradiation. Int J Radiat Oncol Biol Phys 61: 1482-1492, 2005.

9. Kong FM, Ao XP, Wang L and Lawrence TS: The use of blood biomarkers to predict radiation lung toxicity: a potential strategy to individualize thoracic radiation therapy. Cancer Control 15: 140-150, 2008.

10. Novakova-Jiresova A, Van Gameren MM, Coppes RP, Kampinga HH and Groen HJM: Transforming growth factorbeta plasma dynamics and post-irradiation lung injury in lung cancer patients. Radiother Oncol 71: 183-189, 2004.

11. Ao XP, Zhao LJ, Davis MA, Lubman DM, Lawrence TS and Kong FM: Radiation produces differential changes in cytokine profiles in radiation lung fibrosis sensitive and resistant mice. $\mathrm{J}$ Hematol Oncol 2: 6, 2009.

12. Munger JS, Huang X, Kawakatsu H, Griffiths MJ, Dalton SL, Wu J, Pittet JF, Kaminski N, Garat C, Matthay MA, Rifkin DB and Sheppard D: The integrin alpha v beta 6 binds and activates latent TGF beta 1: a mechanism for regulating pulmonary inflammation and fibrosis. Cell 96: 319-328, 1999.

13. Anscher MS, Thrasher B, Rabbani Z, Teicher B and Vujaskovic Z: Antitransforming growth factor-beta antibody 1D11 ameliorates normal tissue damage caused by high-dose radiation. Int J Radiat Oncol Biol Phys 65: 876-881, 2006.

14. Anscher MS: Targeting the TGF-beta1 pathway to prevent normal tissue injury after cancer therapy. Oncologist 15: 350-359, 2010.

15. Sekine I, Sumi M, Ito Y, Nokihara H, Yamamoto N, Kunitoh H, Ohe Y, Kodama T, Saijo N and Tamura T: Retrospective analysis of steroid therapy for radiation-induced lung injury in lung cancer patients. Radiother Oncol 80: 93-97, 2006.

16. O'Reilly MS, Boehm T, Shing Y, Fukai N, Vasios G, Lane WS, Flynn E, Birkhead JR, Olsen BR and Folkman J: Endostatin: an endogenous inhibitor of angiogenesis and tumor growth. Cell 88: 277-285, 1997. 
17. Wang YS, Eichler W, Friedrichs U, Yafai Y, Hoffmann S, Yasukawa T, Hui YN and Wiedemann P: Impact of endostatin on bFGF-induced proliferation, migration, and matrix metalloproteinase-2 expression/secretion of bovine choroidal endothelial cells. Curr Eye Res 30: 479-489, 2005.

18. Ling Y, Lu N, Gao Y, Chen Y, Wang S, Yang Y and Guo QL: Endostar induces apoptotic effects in HUVECs through activation of caspase-3 and decrease of Bcl-2. Anticancer Res 29: 411-417, 2009

19. Zhang L, Ge W, Hu K, Zhang YY, Li CH, Xu XM, He D, Zhao ZY, Zhang JZ, Jie FF, Chen Y and Zheng YF: Endostar down-regulates HIF-1 and VEGF expression and enhances the radioresponse to human lung adenocarcinoma cancer cells. Mol Bio Rep 39: 89-95, 2012.

20. Jia $\mathrm{H}$ and Kling J: China offers alternative gateway for experimental drugs. Nat Biotechnol 24: 117-118, 2006.

21. Ling Y, Yang Y, Lu N, You QD, Wang S, Gao Y, Chen Y and Guo QL: Endostar, a novel recombinant human endostatin, exerts antiangiogenic blocking VEGF-induced tyrosine phosphorylation of KDR/Flk-1 of endothelial cells. Biochem Biophys Res Commun 361: 79-84, 2007.

22. Jiang XD, Dai P, Wu J, Song DA and Yu JM: Inhibitory effect of radiotherapy combined with weekly recombinant human endostatin on the human pulmonary adenocarcinoma A549 xenografts in nude mice. Lung Cancer 72: 165-171, 2011.

23. Wen QL, Meng MB, Yang B, Tu LL, Jia L, Zhou L, Xu Y and $\mathrm{Lu}$ Y: Endostar, a recombined humanized endostatin, enhances the radioresponse for human nasopharyngeal carcinoma and human lung adenocarcinoma xenografts in mice. Cancer Sci 100 $1510-1519,2009$

24. Szapiel SV, Elson NA, Fulmer JD, Hunninghake GW and Crystal RG: Bleomycin-induced interstitial pulmonary disease in the nude, athymic mouse. Am Rev Respir Dis 120: 839-899, 1979.

25. Friedrichs K, Gluba S, Eidtmann H and Jonat W: Overexpression of p53 and prognosis in breast cancer. Cancer 72: 3641-3647, 1993.

26. Jackson IL, Vujaskovic Z and Down JD: A further comparison of pathologies after thoracic irradiation among different mouse strains: finding the best preclinical model for evaluating therapies directed against radiation-induced lung damage. Radiat Res 175: 510-518, 2011.

27. Tsoutsou PG and Koukourakis MI: Radiation pneumonitis and fibrosis: mechanisms underlying its pathogenesis and implications for future research. Int J Radiat Oncol Biol Phys 66: 1281-1293, 2006

28. Liu L, Ding Q, Dai XF, Zhao YX, Ke Y and Wu G: Study on the controlling effect of Shengqi Fuzheng injection on plasma cytokine network in patients with thoracic tumor undergoing radiotherapy. Zhongguo Zhong Xi Yi Jie He Za Zhi 27: 1082-1085, 2007 (In Chinese).
29. Haydont V, Mathé D, Bourgier C, Abdelali J, Aigueperse J, Bourhis $\mathrm{J}$ and Vozenin-Brotons MC: Induction of CTGF by TGF-beta1 in normal and radiation enteritis human smooth muscle cells: Smad/Rho balance and therapeutic perspectives. Radiother Oncol 76: 219-225, 2005.

30. Haydont V, Riser BL, Aigueperse J and Vozenin-Brotons MC: Specific signals involved in the long-term maintenance of radiation-induced fibrogenic differentiation: a role for $\mathrm{CCN} 2$ and low concentration of TGF-beta1. Am J Physiol Cell Physiol 294: C1332-C1341, 2008

31. Franko AJ, Sharplin J, Ghahary A and Barcellos-Hoff MH: Immunohistochemical localization of transforming growth factor beta and tumor necrosis factor alpha in the lungs of fibrosis-prone and 'non-fibrosing' mice during the latent period and early phase after irradiation. Radiat Res 147: 245-256, 1997.

32. Chen L, Brizel DM, Rabbani ZN, Samulski TV, Farrell CL, Larrier N, Anscher MS and Vujaskovic Z: The protective effect of recombinant human keratinocyte growth factor on radiationinduced pulmonary toxicity in rats. Int $\mathrm{J}$ Radiat Oncol Biol Phys 60: 1520-1529, 2004.

33. Folkman J: Antiangiogenesis in cancer therapy-endostatin and its mechanisms of action. Exp Cell Res 312: 594-607, 2006.

34. Itasaka S, Komaki R, Herbst RS, Shibuya K, Shintani T, Hunter NR, Onn A, Bucana CD, Milas L, Ang KK and O'Reilly MS: Endostatin improves radioresponse and blocks tumor revascularization after radiation therapy for A431 xenografts in mice. Int J Radiat Oncol Biol Phys 67: 870-878, 2007.

35. Tanabe K, Maeshima Y, Ichinose K, Kitayama H, Takazawa Y, Hirokoshi K, Kinomura M, Sugiyama $\mathrm{H}$ and Makino $\mathrm{H}$ : Endostatin peptide, an inhibitor of angiogenesis, prevents the progression of peritoneal sclerosis in a mouse experimental model. Kidney Int 71: 227-238, 2007.

36. Vujaskovic Z, Anscher MS, Feng QF, Rabbani ZN, Amin K, Samulski TS, Dewhirst MW and Haroon ZA: Radiation-induced hypoxia may perpetuate late normal tissue injury. Int J Radiat Oncol Biol Phys 50: 851-855, 2001.

37. Gauter-Fleckenstein B, Fleckenstein K, Owzar K, Jiang C, Reboucas JS, Batinic-Haberle I and Vujaskovic Z: Early and late administration of MnTE-2-PyP5 ${ }^{+}$in mitigation and treatment of radiation-induced lung damage. Free Radic Biol Med 48: 1034-1043, 2010.

38. Jackson IL, Chen L, Batinic-Haberle I and Vujaskovic Z: Superoxide dismutase mimetic reduces hypoxia-induced $\mathrm{O}_{2}{ }^{*}$, TGF-beta, and VEGF production by macrophages. Free Radic Res 41: 8-14, 2007 